\title{
Comparative analysis of DFT-vdW vs. Coulomb energies for configurational space of layered cathode material at different delithiation levels
}

\author{
Pavel Zolotarev ${ }^{1,2, *}$ and Roman Eremin ${ }^{1,2}$ \\ ${ }^{1}$ Samara Center for Theoretical Materials Science, Samara University, Samara, Russia \\ ${ }^{2}$ Samara Center for Theoretical Materials Science, Samara State Technical University, Samara, Russia
}

\begin{abstract}
Doping and intercalation ion disordering complicate drastically computer modeling of cathode material properties and behavior during charge-discharge processes. For layered cathode material, parameters of the energetically favored entries of compositional/configurational spaces (CCS) are known to correlate well with the CCS mean values. For the CCS (20760 configurations) of commercially used $\mathrm{Li}_{1-x} \mathrm{Ni}_{0.8} \mathrm{Co}_{0.15} \mathrm{Al}_{0.05} \mathrm{O}_{2}$, a comparative analysis of electrostatic energies and those obtained using first-principles calculations was performed. Based on the Coulomb energy values, the CCS subgroups containing energetically favorable configurations (ca. $25 \%$ of the total number of CCS entries) can be identified reliably for Li deficiencies $x \leq 0.5$. Further delithiation results in discrepancies between the title methods demonstrating a limited applicability of the simplified selection procedure. The most apparent reason for this is dispersion interaction which can significantly influence structure behavior and shift energetic properties of the layered cathode materials at high lithium deintercalation levels.
\end{abstract}

\section{Introduction}

Comprehensive computer modeling approaches applied to investigations of compositional/configurational spaces (CCSs) of existing or hypothetic compounds allows one to distinguish the most relevant and reliable composition-structure-property correlations and, consequently, provides a tool for modifications of material properties, synthesis of new compounds, etc. For example, such correlations can be effectively used to predict the stability and/or behavior of a functional material vs. its chemical composition avoiding long-term synthesis, treatment and experimental measurement for a series of compounds [1]. In other cases, computer modeling can help to reduce significantly a set of promising compounds to be tested experimentally within the task of computational design or searching for new functional materials, e.g., for solid electrolytes [2,3].

\footnotetext{
*Corresponding author: pavelzolotarev@sctms.ru
} 
In our previous work [4], we combined the geometrical-topological approach and the density functional theory calculations with dispersion correction (DFT-vdW) for CCSs sampling and their relaxation for the well-known $\mathrm{LiNiO}_{2}$ (LNO, 87 configurations) and doped $\mathrm{LiNi}_{0.8} \mathrm{Co}_{0.15} \mathrm{Al}_{0.05} \mathrm{O}_{2}$ ( $\mathrm{NCA}, 20760$ configurations) cathode materials. Based on the DFT-vdW results for NCA, it was shown that the structural peculiarities and charge distributions of the most energetically favorable configuration are in a good consistence with the full CCS Boltzmann averages calculated at each delithiation level (among 13 studied). Furthermore, the obtained dependencies qualitatively reproduced the NCA structure behavior studied by means of in situ neutron [5] and X-ray [6] diffraction, as well as oxidation states of transition metals investigated in X-ray absorption fine structure analysis [7].

The aim of the current research is the Coulomb energy approach verification within the comparative analysis of the electrostatic energy values with that of the DFT-vdW approach for the NCA CCS energy balance evaluation. In case of a strong correlation between DFTvdW and Coulomb energies, the most energetically favored configurations can be selected at the CCS setting stage and studied within the comprehensive modeling approaches. Thus, the efficiency of reducing the number of independent configurations in the proposed scheme is additionally investigated within the current research.

\section{Methods}

The Coulomb energy calculations were performed using the Supercell program [8] for each independent configuration of the NCA CCS (20760 structures) and compared with the previous DFT-vdW results [4]. The corresponding charge distributions (ion oxidation states) among the model cell were chosen as follows: 1) $\mathrm{Li}, \mathrm{O}$, and $\mathrm{Al}$ ions have constant oxidation states of $+1.0,-2.0$, and +3.0 , respectively (all values are the units of the electron charge absolute value); 2) $\mathrm{Ni}$ and Co ions change the oxidation states linearly in the range from +3.0 to +4.0 to fit the electroneutrality of the model cell under the composition changes in delithiated states. Thus, in a full accordance with the developing approach, the mentioned rules can be a priori chosen.

The obtained energy values (after normalization to the numbers of atoms in the model cell) were compared to that of the DFT-vdW approach. The criterion for successful energy forecasting is an assignment of favorable configuration (DFT-vdW) to a group of structures with minimal electrostatic interaction energies. The above-mentioned groups of configurations naturally arise due to the principal indistinguishability of the CCS entries with respect to $\mathrm{Co} / \mathrm{Ni}$ permutations within the Coulomb energy calculations.

\section{Results}

Obviously, for the NCA cathode material $\left(\mathrm{Li}_{1-x} \mathrm{Ni}_{0.8} \mathrm{Co}_{0.15} \mathrm{Al}_{0.05} \mathrm{O}_{2}\right)$ the Coulomb energies of the fully intercalated $(\mathrm{x}=0)$ configurations have the same value due to the equal charge values for $\mathrm{Ni}, \mathrm{Co}$ and $\mathrm{Al}$. In this case, all configurations should be considered at the following stages. Comparison of the DFT-vdW vs. Coulomb energies is shown in Fig.1 and Fig. 2 for the low/low-medium and high-medium/high delithiation levels, respectively. The 
configuration splitting in accordance with their Coulomb energies can be distinguished as sharp vertical sets of points in Fig.1 and Fig.2.
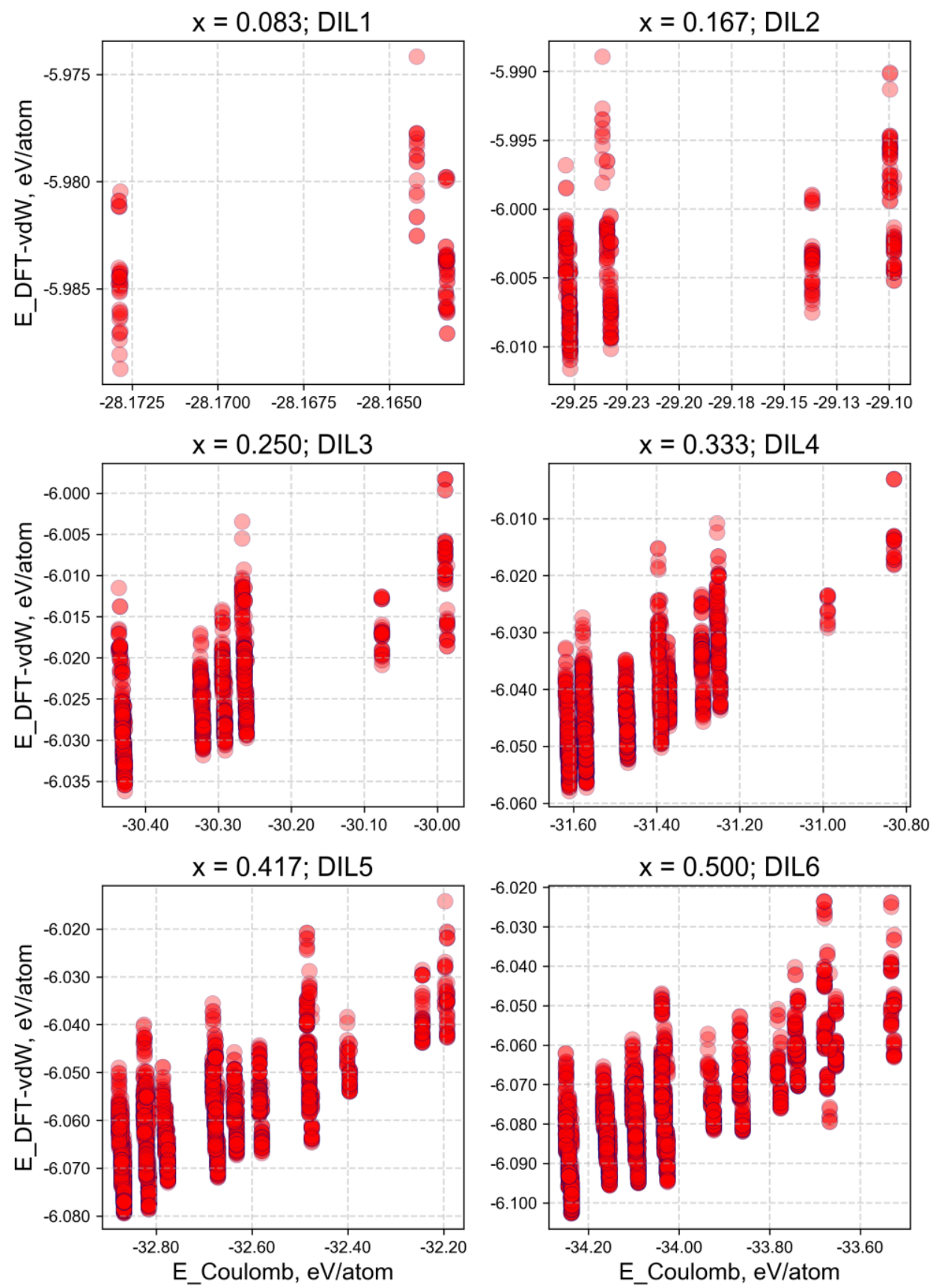

Fig. 1. Comparison of the DFT-vdW $\left(E_{-} D F T-v d W\right)$ and Coulomb $\left(E_{-}\right.$Coulomb) energies for the NCA CCS subset corresponding to low and medium-low DILs: $\mathrm{Li}_{1-\mathrm{x}} \mathrm{Ni}_{0.8} \mathrm{Co}_{0.15} \mathrm{Al}_{0.05} \mathrm{O}_{2}$, where $0<x \leq 0.5$. 

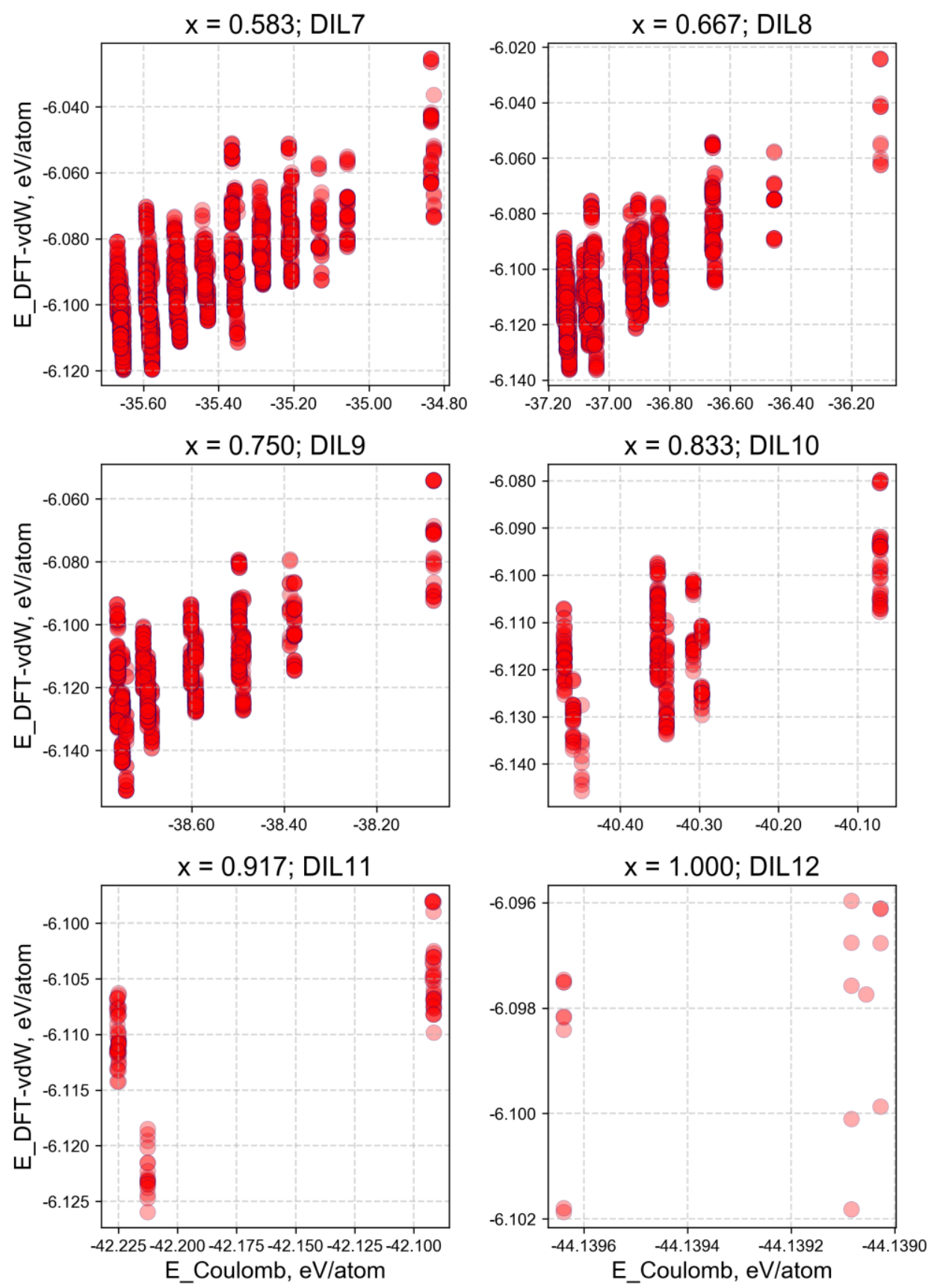

Fig. 2. Comparison of the DFT-vdW (E_DFT-vdW) and Coulomb (E_Coulomb) energies for the NCA CCS subset corresponding to medium-high and high DILs: $\mathrm{Li}_{1-x} \mathrm{Ni}_{0.8} \mathrm{Co}_{0.15} \mathrm{Al}_{0.05} \mathrm{O}_{2}$, where $0.5<x \leq 1$.

The results for $0<x \leq 0.5$ allow one to conclude that the Coulomb energy-based approach predictions satisfy the introduced 'success' criterion for the considering deintercalation levels (DILs), the relative energy differences (RED) for the configurations 
within the most energetically favored groups (by means of the Coulomb energy approach) do not exceed $0.03 \%$. Whereas, the RED values for the energetically favored groups and the nearest neighbor groups are in the range of $0.04-0.20 \%$. As a result, the CCS complexity can be reduced by a factor of ca. 4 (27\% configurations) using the Coulomb energy-based selection. For $x=0.583$ and 0.667 , the lowest energy configurations (according to the DFT-vdW approach) are in two different Coulomb energy groups. For the further delithiation $(x>0.75)$ levels, the tested Coulomb energy-based assessment results in a wrong prediction of the lowest-energy subset.

\section{Conclusions and remarks}

For the doped LNO family cathodes, the performed comparative analysis demonstrates the limited applicability of the Coulomb energy estimations for selection of the energetically favored configurations. For deintercalation levels up to $x=0.5$, the Coulomb energy-based approach allows to reliably select the lowest energy CCS entries. On the other hand, the introduced criterion of successful energy estimation is not satisfied at the higher delithiation levels. Based on the results of DFT calculations $[9,10]$, the apparent reason for such observation is dispersion interaction which can contribute significantly to the structure energies at high delithiation. The latter causes significant structural changes of the layered cathode materials altering the DFT-vdW energies with respect to that calculated within the electrostatic approach for the non-optimized NCA CCS. It is worth noting, that Coulomb energy-based selection of the energetically favored entries allowed to reduce the CCS complexity by a factor of ca. 4 in the deintercalation range of $0<x \leq 0.5$. Thus, the reduced CCS is comparable in complexity with that obtained within the recently proposed machine learning, DFT calculations, and geometrical-topological analysis combined approach [11] remaining applicable in the whole range of delithiation.

We are obliged to computational facilities of the 'Zeolite' supercomputer of Samara Center for Theoretical Materials Science (Samara University). The study was supported by the Ministry of Education and Science of the Russian Federation (project No.3.6588.2017/9.10).

\section{References}

1. Urban A., Matts I. et al. Adv. Energy Mater. 6, 1600488 (2016).

2. Anurova N.A., Blatov V.A. et al. Solid State Ionics 179, 2248-2254 (2008).

3. Meutzner F., Münchgesang W. Chem. - A Eur. J. 21, 16601-16608 (2015).

4. Eremin R.A., Zolotarev P.N. et al. J. Phys. Chem. C 121, 28293-28305 (2017).

5. Bobrikov I.A., Samoylova N.Yu. et al. J. Power Sources 372, 74-81 (2017).

6. Robert R., Bünzli C. et al. Chem. Mater. 27, 526-536 (2015).

7. Nakai I., Nakagome T. Electrochem. Solid-State Lett. 1, 259-261 (1998).

8. Okhotnikov K., Charpentier T., Cadars S. J. Cheminform. 8:17 (2016).

9. $\quad$ Eremin R., Zolotarev P., Bobrikov I. EPJ Web Conf. 177, 02001 (2018).

10. Aykol M., Kim S., Wolverton C. J. Phys. Chem. C. 119,19053-19058 (2015).

11. Zolotarev P., Eremin R. EPJ Web Conf. 177, 02005 (2018). 